\title{
On the acquisition and measurement of stimulus control in pigeons
}

\author{
ROBERT J. NEWLIN and DAVID R. THOMAS \\ University of Colorado, Boulder, Colorado
}

\begin{abstract}
Two experiments were performed to investigate the relationship between excitatory stimulus control (number of responses to a training stimulus) and dimensional stimulus control (generalization gradient slope). In experiment 1 , after being trained to peck a green key, pigeons received either 20,40 , or 80 brief $(.5,2,4$, or $8 \mathrm{sec})$ presentations of a 45-deg line followed by reinforcement (12 groups) or 20,40, or 80 reinforcements for pecking a continuously presented 45-deg line (3 groups). Number of reinforcements determined the slope (percent of total responses to $45 \mathrm{deg}$ ) of a subsequent line-angle generalization gradient, but number of responses to the 45deg line in the test was controlled by total experience with $45 \mathrm{deg}$ as measured by either total exposure time or total responses to $45 \mathrm{deg}$ in training. In a second experiment, it was shown that increasing the number of days of pretraining to green decreased the slope of the gradient (in subjects given 2-sec presentations), but had no effect on number of responses to $45 \mathrm{deg}$ in the test. Furthermore, continuous presentation yielded flatter gradients but more responding to the 45-deg line in the test than did 2-sec presentations. It was concluded that the measures of dimensional stimulus control and excitatory stimulus control reflect different processes because they vary differentially (sometimes in different directions) in response to the same independent variable manipulations.
\end{abstract}

The concept of stimulus control is discussed in most contemporary textbooks on learning, but the term is used in two different ways. One of these, called "excitatory stimulus control" by Hearst, Besley, and Farthing (1970), refers to the extent to which a specified response is more probable in the presence of a stimulus than in its absence. For practical purposes, a measure of response strength (e.g., rate or magnitude) provides an index of excitatory stimulus control. This measure has been used extensively in the study of classical conditioning. Alternatively, "dimensional stimulus control," according to Hearst et al., refers to the slope of a stimulus generalization gradient obtained by varying the training stimulus along some physical continuum (e.g., wavelength or intensity). The measure of relative generalization slope (e.g., the percentage of all responses during the generalization test which occur to the training stimulus) is frequently used in operant conditioning studies. It is commonly assumed, either explicitly or implicitly, that excitatory and dimensional stimulus control are two measures of the same learning process (cf. Blough, 1975; Mackintosh, $1975,1977)$, but this is an empirical question that has rarely been addressed. Confirmatory evidence would

This research was supported by NSF Grant BNS 78-01407 to David R. Thomas. It was presented at the April 1981 meeting of the Rocky Mountain Psychological Association in Denver. Requests for reprints should be addressed to: David R. Thomas, Department of Psychology, Campus Box 345, University of Colorado, Boulder, Colorado 80309. consist of a positive correlation between the two measures and the determination that they are similarly affected by the same controlling variables, that is, amount of training, amount of reinforcement, etc.

The two measures of stimulus control are positively correlated under some conditions. Hearst and Koresko (1968) obtained line-orientation stimulus generalization gradients in pigeons after 2 to 1450 min sessions of variable-interval (VI) 1-min training. Both response rate to the training stimulus and relative gradient slope increased with increased training. Schadler and Thomas (1972) attempted to separate acquisition of the response from acquisition of stimulus control by pretraining pigeons to peck a white key for 1030 -min sessions of VI 1-min reinforcement and then giving $0,5,10$, or $20 \mathrm{~min}$ of VI 1-min training with a line. With this procedure, the slope of the angularity gradients increased with increased line angle training, whereas overall response rate did not change significantly. Pigeons with 0 and $5 \mathrm{~min}$ of training responded less to the training stimulus than did pigeons given 10 or 20 min of training, but this difference was not tested for significance. In a further study with this procedure, Weisman and Thio (1976) trained pigeons to peck a line and then gave varying amounts of training to a color reinforced on either a VI 30-sec schedule or a VI 10-sec one. For both schedules, during testing on wavelength, both overall rate of responding and relative gradient slope increased as a function of amount of training, but the correlation between the two was low across conditions $(r=.09, F<1.0)$. Since steeper gradients reflect 
relatively more responding to the training stimulus, one would expect that the correlation between slope and responding to the training stimulus would be somewhat larger. Further analysis indicated that relative gradient slope was a function of number of reinforcers rather than duration of training. Unfortunately, no parallel analysis was done with either overall rate of responding or responding to the training stimulus.

Apart from the study of acquisition of stimulus control, the relationship between dimensional and excitatory measures has been more variable. For example, Hearst and Poppen (1965) reported that administering a period of extinction of operant responding to a training stimulus reduced the level of responding in a subsequent generalization test while sharpening the relative generalization gradient. Thomas and King (1959) trained different groups of subjects to peck at a given color while at $80 \%$ of adlib weight and then tested them for wavelength generalization, after a 1-week delay, at $60 \%, 70 \%, 80 \%$, or $90 \%$ of ad-lib weight. Relative generalization slope, that is, dimensional stimulus control, was nonmonotonically related to drive level, being greatest at $80 \%$, lower at $70 \%$ and $90 \%$, and lowest at $60 \%$. The relative generalization gradients of $70 \%$ and $90 \%$ groups were indistinguishable despite the fact that the $70 \%$ group responded almost four times as much to the training value. Furthermore, analysis of the relative generalization gradients of the three highest and three lowest responders within each deprivation group showed no differences.

The purpose of the present study was to explore further the measures of excitatory and dimensional stimulus control during the course of acquisition in order to determine whether two different learning processes were involved. This was accomplished by comparing the two measures while introducing a variety of procedural manipulations. If it was the number of reinforcements which most directly determined gradient slope, as suggested by Weisman and Thio (1976), then comparable gradients should be obtained with the typical procedure of presenting the training stimulus continuously and an alternative procedure in which the training stimulus is presented briefly prior to the delivery of a reinforcement. In the present study, pigeons were trained to peck a green key on a VI 1 -min schedule. In most groups, after several sessions the schedule was changed such that, instead of immediate reinforcement, a peck produced a brief $\left(45^{\circ}\right.$ line) stimulus on the key, which was followed by reinforcement. This procedure allowed us to control accurately both the duration of exposure to the line and the number of reinforcements obtained in its presence. Angularity generalization gradients obtained with this procedure were compared with those obtained using a continuous presentation of the line angle training stimulus.

\section{EXPERIMENT 1}

\section{Method}

Subjects. The subjects were 216 experimentally naive adult pigeons obtained from a local supplier. They were maintained at approximately $75 \%$ of their ad-lib weights in a colony with a $16-\mathrm{h}$ light/8-h-dark cycle. Sessions were conducted between 1 and $11 \mathrm{~h}$ after the lights were switched on.

Apparntus. Four standard single-key conditioning chambers (cf. Hickis, Robles, \& Thomas, 1977) were used. On-key stimuli were provided by IEE (Series 0010 ) in-line display cells mounted $.635 \mathrm{~cm}$ behind the key. Kodak Wratten filter No. 99 provided the green $(555 \mathrm{~nm})$ stimulus. The display cells also allowed projection of white lines, $.2 \mathrm{~cm}$ wide and $2.5 \mathrm{~cm}$ long, on a dark surround at angles of 9, 27, 45, 63, and $81 \mathrm{deg}$ from horizontal. Chamber illumination during all sessions was provided by a $28-\mathrm{V}$ miniature lamp (No. 1829) centered behind a 2.4-cm-high strip of translucent white Plexiglas extending the width of the chamber above the intelligence panel.

Procedure. On Day 1, all pigeons were hand-shaped to peck the green key for $3 \mathrm{sec}$ of access to mixed grain and allowed to earn 60 reinforcements on a gradually increasing VI schedule. On Day 2 , all pigeons were allowed to earn 50 reinforcements on a gradually increasing VI schedule. For the next 10 days, all pigeons earned 30 reinforcements per day on a VI 1-min schedule. On the next day, all subjects earned 20 reinforcements on the VI 1 -min schedule and then received a line-angle generalization test in extinction.

As described below, for most subjects on some predetermined number of occasions when a food reinforcement was earned via a peck at the green key, a 45-deg white line was presented (on a dark background) for a brief period prior to the activation of the food hopper. The last 20, 40, or 80 reinforcements earned during training (on the last, last 2 , or last 3 days, respectively) were preceded by .5-, 2-, 4-, or 8-sec-duration presentations of the 45 -deg line in different groups (12 groups). Three other groups were allowed to earn their last 20,40 , or 80 reinforcements by pecking the 45 -deg line, which was presented continuously, giving them 20,40 , or 80 min experience, respectively, with it. These latter groups therefore received the Schadler and Thomas (1972) procedure. Seven subjects which failed to maintain pecking when the line stimulus was introduced were replaced.

Each subject received one of eight different generalization tests consisting of seven blocks, during each of which each of the five line-angle stimuli was presented once (for $20 \mathrm{sec}$ ) in random order. The first block was not used in the analysis because responding during the first 10 to $30 \mathrm{sec}$ of the test was found to be extremely high, thus yielding gradients for individual subjects that depended on which stimulus was presented first in the test. Another consequence of the training procedure was the rapid extinction of responding in some subjects. In order to permit the calculation of a reliable measure of dimensional stimulus control (percent of total responses to each stimulus), subjects that failed to respond at least 20 times and in at least two of the last six blocks were replaced until 12 subjects in each group had met this criterion. Table 1 lists the conditions and the number of subjects failing to reach criterion in each condition.

\section{Results and Discussion}

In this experiment, a moderate, but significant, positive correlation was found between gradient slope (percent to $45 \mathrm{deg}$ ) and responses to $45 \mathrm{deg}$ during the test $[r=.20 ; F(1,178)=7.34, p<.01]$. The correlation between gradient slope and total responses during the test was essentially zero $[r=-.08$; $F(1,178)=1.08, p>.30]$. Having established a small positive correlation between the measures of excitatory and dimensional-stimulus control, we turn now to an analysis of the factors controlling each. 
Table 1

Conditions Employed in Experiment 1

\begin{tabular}{|c|c|c|c|c|c|}
\hline \multirow[b]{2}{*}{$\begin{array}{c}\text { Number of } \\
\text { Reinforcements }\end{array}$} & \multirow[b]{2}{*}{$\begin{array}{l}\text { Length of } \\
\text { Presentation* }\end{array}$} & \multirow[b]{2}{*}{$\begin{array}{c}\text { Total } \\
\text { Exposure* }\end{array}$} & \multicolumn{3}{|c|}{ Number of Subjects } \\
\hline & & & Total & $\begin{array}{c}\text { That Stopped Pecking } \\
\text { Before Test }\end{array}$ & $\begin{array}{l}\text { That Failed to } \\
\text { Meet Test Criterion }\end{array}$ \\
\hline 20 & .5 & 10 & 24 & 1 & 11 \\
\hline 40 & .5 & 20 & 20 & 0 & 8 \\
\hline 80 & .5 & 40 & 12 & 0 & 0 \\
\hline 20 & 2 & 40 & 13 & 1 & 0 \\
\hline 40 & 2 & 80 & 13 & 1 & 0 \\
\hline 80 & 2 & 160 & 14 & 0 & 2 \\
\hline 20 & 4 & 80 & 13 & 0 & 1 \\
\hline 40 & 4 & 160 & 13 & 0 & 1 \\
\hline 80 & 4 & 320 & 13 & 0 & 1 \\
\hline 20 & 8 & 160 & 12 & 0 & 0 \\
\hline 40 & 8 & 320 & 15 & 2 & 1 \\
\hline 80 & 8 & 640 & 15 & 0 & 3 \\
\hline 20 & $\mathrm{C}$ & 1200 & 14 & 1 & 1 \\
\hline 40 & $\mathrm{C}$ & 2400 & 13 & 1 & 0 \\
\hline 80 & $\mathrm{C}$ & 4800 & 12 & 0 & 0 \\
\hline
\end{tabular}

Note-C $=$ continuous. $\quad *$ In seconds.

Percent of responses to the training stimulus is presented in Figure 1. It is clear that, in general, increments in number of presentations (or reinforcements) increased the percentage of responses to $45 \mathrm{deg}$. On the other hand, the shapes of the curves are not orderly, suggesting that changes in duration of presentation did not have a systematic effect. Analysis of variance confirms this impression. A set of comparisons was used which allowed analysis of the linear effect of the levels of each variable (as a conservative test of order) and comparison of the brief and continuous presentation procedures. The (linear) effect of number of presentations was significant $[F(1,165)=8.68, p<.01]$; however, the linear effect of the four durations of presentations was not $[F(1,165)=2.16, p>.10]$. There were no effects for higher order components $(F s<1.0)$. Since the linear effect of duration is a conservative measure, it should be noted that no contrast would have been significant $[F(1,165) \max =3.04, p>.05]$. There was no interaction between duration and number of presentations $(F<1.0)$. Although it would appear that the 40 and 80 continuous-presentation groups' gradients were flatter than those of the corresponding brief presentation groups, the overall effect of procedure was not significant $[F(1,165)=1.74, p>.10]$ and there was no interaction between method of presentation (i.e., procedure) and number of presentations $(F<1.0)$. Thus, our conclusion, similar to that of Weisman and Thio (1976), is that slope of the gradient is determined by the number of reinforcements (or presentations) of the training stimulus. Reliable gradients can be achieved with very little training with the test stimulus; certainly fewer than 40 reinforcements and less than $3 \mathrm{~min}$ of total exposure to the stimulus are needed when using the brief presentation procedure.
It should be noted that, since total responses and gradient slope were not correlated, rejection of those subjects with the least responses should not have biased this result.

The measure of responses to $45 \mathrm{deg}$ is presented in Figure 2. As can be seen, there is a large effect for duration of presentation, with a tendency for those subjects receiving 20 presentations to respond less than those receiving either 40 or 80 presentations. Analysis of variance confirms a (linear) effect of number of presentations $[F(1,165)=7.69, p<.01]$ and of duration $[\mathrm{F}(1,165)=18.153, \mathrm{p}<.01]$. The second-order effect of number of presentations does not reach significance $[F(1,165)=3.16, p>.05]$, nor do the higher order effects of duration (Fs $<1.0$ ).

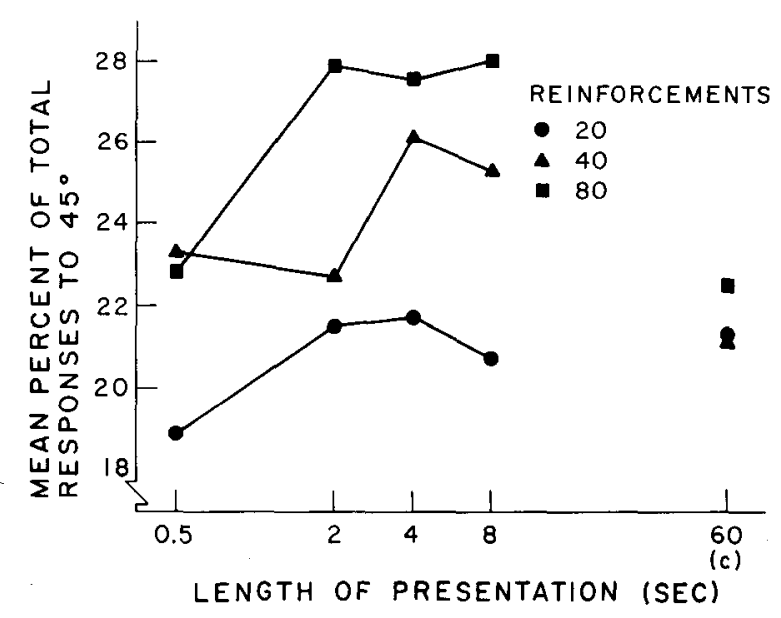

Figure 1. Mean percent of total responses in the test to the 45 deg line as a function of the length of presentation or continuous presentation procedure (c) in training for groups receiving 20,40 , and 80 reinforcements in the presence of the $45-\mathrm{deg}$ line in training. 


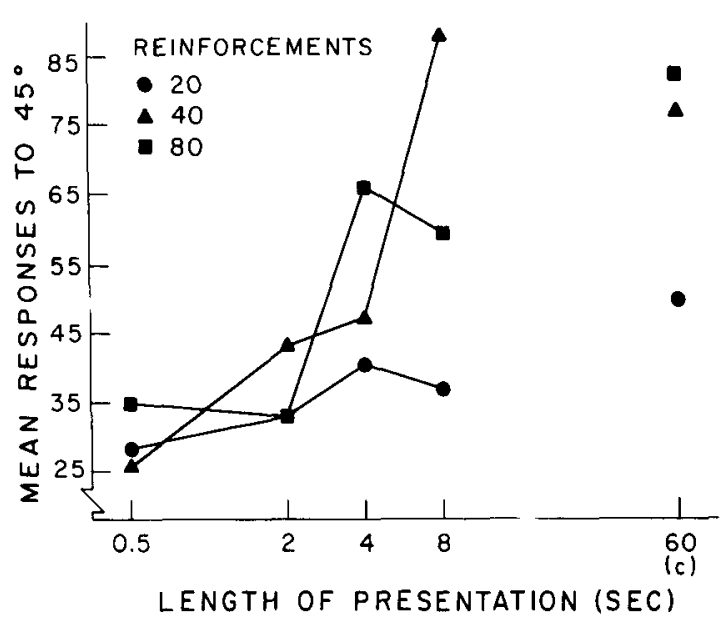

Figure 2. Mean responses to the 45-deg line in test as a function of the same training variables as in Figure 1.

There is no interaction between number and duration of presentations $[F(6,165)=1.89, p>.05]$. There is an effect of procedure with continuous-presentation subjects responding more than those given brief presentations $[F(1,165)=14.93, p<.01]$. There is no interaction between procedure and number of presentations (or reinforcements) $(F<1.0)$. The conclusion is that number of presentations, duration of presentation, and procedure all had effects on number of responses to $45 \mathrm{deg}$. It should be noted that since responding to $45 \mathrm{deg}$ is highly correlated with total responding $(r=.95)$, rejection of the subjects with low rates leads to an underestimate of the effects on responding to $45 \mathrm{deg}$.

In the above analysis, it was shown that responding to $45 \mathrm{deg}$ increased as presentation duration increased, as number of presentations increased, and as the procedure was changed from the brief to the continuous presentation procedure. All three of these factors have in common an increase in total exposure time to the stimulus. Figure 3 presents the mean number of responses to the 45 -deg line in the test as a function of total exposure time to $45 \mathrm{deg}$ in training plotted on a log scale. For this figure and the following analysis, the data from all 209 subjects tested were used. The correlation between responses to $45 \mathrm{deg}$ and log total exposure time is .46 $[\mathrm{F}(1,207)=$ 55.94, $\mathrm{p}<.01]$. As total exposure time increases, so does responding to the 45-deg line during training $(r=.92)$, and the number of responses to 45 deg during training is also highly correlated with number of responses to $45 \mathrm{deg}$ during test $[r=.52 ; F(1,207)=$ $75.86, p<.01]$. Thus, it seems likely that the factor which contributes most strongly to excitatory stimulus control is total experience with the stimulus; however, further experiments using this factor explicitly as an independent variable should be conducted to establish this conclusion more firmly.
To summarize, it would appear that sharpness of the gradient, that is, dimensional stimulus control, is determined by the number of reinforcers obtained in the presence of the stimulus, while amount of responding to the stimulus in test, that is, excitatory stimulus control, is determined by amount of total experience with the stimulus.

Before turning to a further experiment, one other aspect of these data deserves attention. By using a 45deg line and testing symmetrically around it, we can examine a further finding reported by Schadler and Thomas (1972). These investigators found that pigeons without experience in pecking lines but with training in pecking a white key light tended to peck the vertical $(90 \mathrm{deg})$ line more often than they did lines of 60 or $30 \mathrm{deg}$. This initial preference was overcome with reinforced training with the 30-deg line. In the present experiment, neither vertical nor horizontal lines were present in the test; $9,27,45,63$, and $81 \mathrm{deg}$ were used. However, it was possible to ask whether pigeons tended to peck more to stimuli oriented closer to vertical than to horizontal and to determine whether this preference, if it existed, was affected by increased training to the $45-\mathrm{deg}$ value. The birds did show a preference for stimuli on the vertical side of $45 \mathrm{deg}$, with the mean of the percent of all responses to these stimuli being 40.5 and the mean of the percent of responses to stimuli on the horizontal side of $45 \mathrm{deg}$ being 36.1. Despite the small size of this difference, the effect was quite consistent so that analysis of variance showed it to be significant $[F(1,65)=8.04, p<.01]$ and there was no interaction with either number of presentations $[\mathrm{F}(2,162)=1.49$, $p>.20]$ or length of presentation $[F<1.0]$ or threeway interaction $[F(8,165)=1.95, p>.05]$. Thus, stimuli closer to vertical are pecked more than are stimuli closer to horizontal, but experience with $45 \mathrm{deg}$ does not seem to affect that preference, at

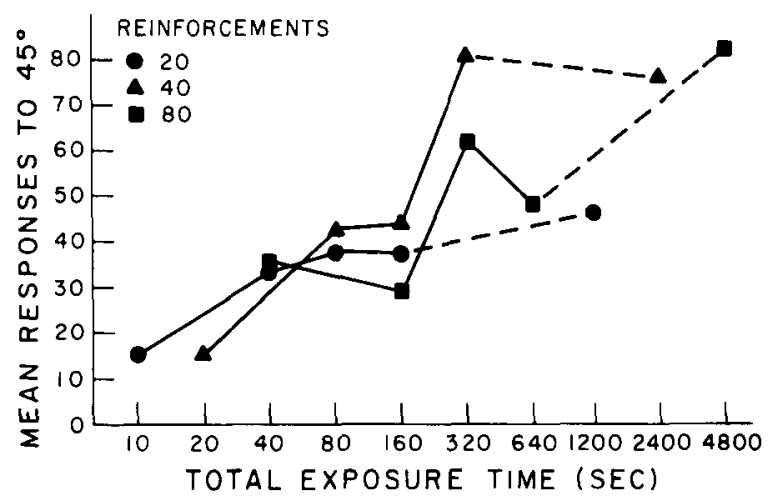

Figure 3. Mean responses to the 45-deg line in test as a function of total exposure time (log scale) in training for groups receiving 20,40 , or 80 reinforcements in the presence of the 45 -deg line in training. 
least within the range of 20 reinforcements and $10 \mathrm{sec}$ to 80 reinforcements and $80 \mathrm{~min}$ of total experience.

\section{EXPERIMENT 2}

Schadler and Thomas (1972) attempted "to isolate the acquisition of dimensional control from response learning"' (p. 82). They felt that the increase in both steepness of gradient and amount of responding found by Hearst and Koresko (1968) were confounded between learning to peck in the situation and experience with the stimulus dimension being tested. They reasoned that when separated from the acquisition of responding, the acquisition of dimensional stimulus control might be shown to be much more rapid than previously suspected. Furthermore, it is possible that learning to peck might interfere with learning what to peck at, such that the more pretraining that was given, and thus the better learned the pecking response, the more rapid the acquisition of dimensional stimulus control might be. In Experiment 2 , we investigated this possibility by replicating the 40 reinforcement groups given either continuous stimulus presentation or 2-sec presentation while decreasing pretraining by half in one-third of the pigeons and doubling it in another third.

This procedure allows us to look again at the difference between brief and continuous presentation. For the 40 and 80 reinforcement groups of Experiment 1 , the gradients produced by continuous presentation appeared to be much flatter than those produced by brief presentation. This effect did not reach significance, possibly due to a floor effect in the 20 presentation groups ( $20 \%$ represents equal responding to all stimuli and can be considered the floor for discrimination).

\section{Method \\ Subjects. The subjects were 80 experimentally naive adult pi- geons maintained as in Experiment 1. \\ Procedure. The procedure was the same as that in Experiment 1 for the 40 reinforcement 2 -sec and continuous presentation groups, with two exceptions. First, there were only 10 reinforce- ments on the test day. Second, training consisted of 5, 10, or 20 sessions for different groups rather than the 10 sessions used in Experiment 1. As before, pigeons that failed to peck at least 20 times and in at least two of the last six blocks during the test were replaced. Eight pigeons were replaced, with no more than three in any group. This design yielded six groups (2-sec or continuous pre- sentation by 5,10 , or 20 sessions training) with 12 subjects per group.}

\section{Results and Discussion}

The change in pretraining was successful in modifying responding to the green key. On the last day on which the green key alone was presented, mean number of responses increased reliably from 1,202 in the 5-day groups to 1,375 in the 10-day groups to 1,601 in the 20-day groups $[F($ linear $)(1,66)=8.92, p<.01]$. There was no difference between groups that received continuous and brief presentations and no interaction $(\mathrm{Fs}<1.0)$.

During testing, the correlation between percent of responses to $45 \mathrm{deg}$ and number of responses to $45 \mathrm{deg}$ was essentially zero $(r=.04 ; F<1.0)$.

Figure 4 presents the percent of responses to $45 \mathrm{deg}$ for both 2 -sec and continuous presentation groups as a function of days of training. As can be seen, there is a large decrease in sharpness of gradient for the 2-sec presentation groups as days of pretraining increase. The continuous presentation groups all show relatively flat gradients regardless of the amount of pretraining. Analysis of variance confirms a decrease in percent of response to $45 \mathrm{deg}$ with increasing levels of training $[F($ linear $)(1,66)=5.22, p<$ $.05]$ and a difference between 2 -sec and continuous presentation $[F(1,66)=7.64, p<.01]$, as well as a significant (linear) interaction $[F(1,66)=4.32$, $\mathrm{p}<.05]$. Thus, length of pretraining affects gradient slope, at least in the 2 -sec presentation procedure, with increasing pretraining on green yielding flatter gradients on line angle. The lack of a parallel effect in the continuous presentation groups may be due to a floor effect, since even with only 5 days of pretraining these gradients were essentially flat.

In view of the negative correlation between amount of pretraining and gradient slope, it is clear that learning of the keypecking response does not interfere with the acquisition of dimensional control by the 45-deg training stimulus. In fact, continued keypeck training interferes with that acquisition process. Furthermore, continuous presentation of the stimulus leads to flatter gradients than $2-\mathrm{sec}$ presentations even though all groups received 40 reinforcements in the presence of the 45-deg line and continuous presentations groups had more experience with the stimulus.

Figure 5 presents the responses to $45 \mathrm{deg}$ during

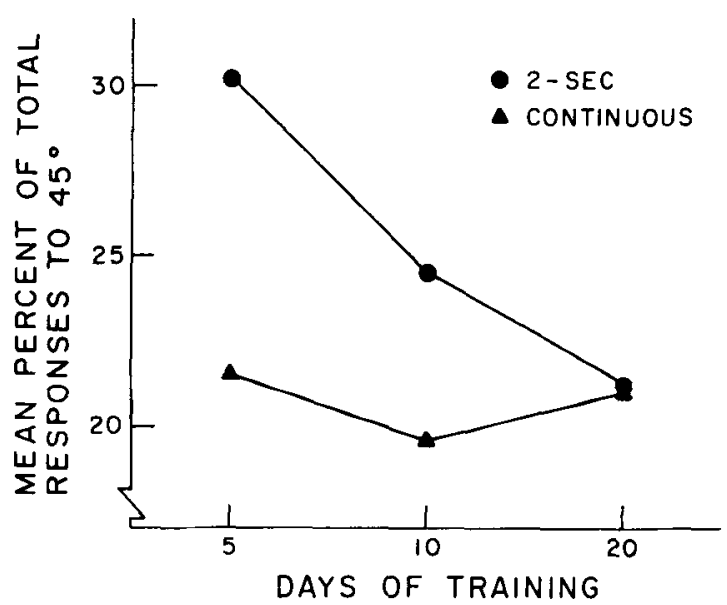

Figure 4. Mean percent of total responses in the test to the 45deg line as a function of length of pretraining for groups that received 40 reinforcements with either the brief $(2-\mathbf{s e c})$ or the continuous presentation procedure. 
testing as a function of amount of pretraining. As can be seen, both groups responded least to $45 \mathrm{deg}$ after 10 days of training and most after 20 days, but these differences were not significant $[F($ linear $)(1,66)=$ $2.89, \mathrm{p}>.05 ; \mathrm{F}$ (quadratic) $(1,66)=1.65, \mathrm{p}>.20$ ] However, there was a big difference in responding between continuous and 2 -sec presentation procedures, with the continuous groups responding more, as in Experiment $1[\mathrm{~F}(1,66)=14.70, \mathrm{p}<.01]$. There was clearly no interaction between days of pretraining and presentation procedure $(F<1.0)$.

Thus, a variable, number of days of pretraining, which is very effective in modulating dimensional stimulus control, is ineffective in changing excitatory stimulus control. Another variable, continuous vs. 2 sec presentation, affects the two measures in opposite directions.

The question of what the differences are in the brief and continuous presentation procedure that yield these opposite effects on the two measures of stimulus control must be considered. The brief presentation procedure can be thought of as an autoshaping schedule embedded in an operant schedule. Thus, the 45-deg line in the brief presentation procedure is more predictive of reinforcement than it is in the continuous procedure. In autoshaping, the more predictive stimulus should lead to more pecking (more excitatory control), with continuous presentation yielding no pecking (cf. Schwarz \& Gamzu, 1977). However, our results are in the opposite direction. Thus, continuous presentation led to more pecking than did brief exposures, and, within the brief presentation procedure in Experiment 1, in which the shorter presentations are presumably more predictive than the longer ones, they led to less pecking in the test rather than more. In terms of dimensional control, the brief presentation procedure led to

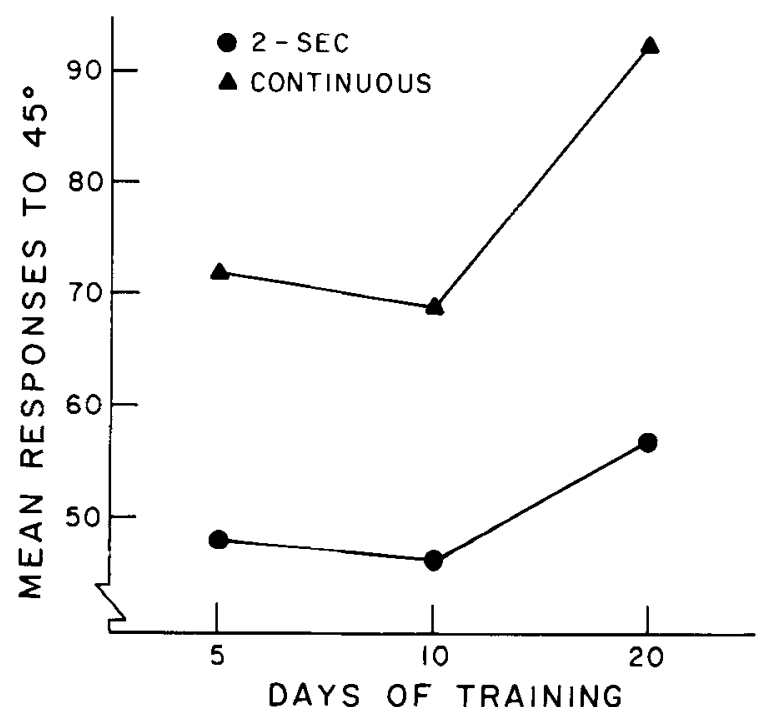

Figure 5. Mean responses to the 45-deg line in test as a function of the same training variables as in Figure 4. more control, but the length of presentation was not effective in modulating that control.

Weisman and Thio (1976) found that pigeons that had had prior discrimination training on line angle, produced sharper wavelength gradients after $4 \mathrm{~min}$ of VI 30-sec training on green than did birds without discrimination training. It seemed plausible that the brief presentation procedure in the present study might have acted like discrimination training between the 45-deg line $(\mathrm{S}+)$ and green $(\mathrm{S}-)$, thus leading to sharper gradients. To test this possibility, as well as to provide an additional training manipulation which might affect the two stimulus control measures differentially, in an additional experiment six groups of 16 pigeons were given the 10 -day, 40 -reinforcement, 2 -sec (three groups) and continuous (three groups) procedures of Experiment 2. For four groups (two in each procedure), Days 3 through 6 of training consisted of discrimination training between green $(555 \mathrm{~nm})$ and red $(606 \mathrm{~nm})$ or green $(555 \mathrm{~nm})$ and green $(538 \mathrm{~nm})$. The results gave no indication of an effect for discrimination training; thus, this manipulation provided no additional information on the relationship between the two measures of stimulus control. This experiment did, however, replicate the differences between brief and continuous exposure procedures found in Experiments 1 and 2. The continuous procedure produced flatter gradients than the brief exposure procedure $[F(1,90)=7.19, p<.01]$. Furthermore, the continuous presentation groups responded more to 45 deg than did the brief presentation groups $[F(1,90)=6.09, p<.02]$. Whether or not additional days of discrimination training would have led to a measurable effect is, of course, an empirical question, but it was not pursued, since it is not central to our concern about the independence of the two stimulus control measures.

\section{GENERAL DISCUSSION}

It was not our purpose to determine the minimal amount of stimulus exposure and/or number of reinforcers necessary for the acquisition of dimensional stimulus control. That value must depend on many factors, including the number of subjects employed and the dimensional separation of the stimuli presented in the test. Clearly, if dimensional stimulus control is the objective, the discrete trial procedure yields steeper gradients with fewer reinforcements and much less total exposure to the stimulus. However, if excitatory stimulus control is the objective, the continuous presentation procedure will yield more responding after fewer reinforcements, but excitatory control is a function of total exposure time regardless of the procedure.

The positive correlation found between gradient slope and amount of responding by Hearst and Koresko (1968), Weisman and Thio (1976), and in 
Experiment 1 of this paper is apparently the result of a positive correlation between amount of experience with the stimulus and number of reinforcements presented during the stimulus. Although most experimental designs have probably included the positive correlation between experience and reinforcement found in the Hearst and Koresko (1968) experiment, the results of other experiments, including the present study, should make it clear that a theory of stimulus control based on either excitatory or dimensional stimulus control will not be generalizable to the other measure under all conditions. Note, however, that Blough (1975) and Mackintosh (1975, 1977) explicitly assume that the two measures reflect a single unitary process. The same assumption is implicitly made by many others. For example, Nevin (1973) and Schwartz (1978) never explicitly state how stimulus control is to be measured. Although both describe in detail the use of gradients to measure stimulus control, when describing the variables affecting stimulus control, they cite both experiments using gradients and experiments using rate of responding as the dependent variable. The problem that this creates can be illustrated by using the phenomenon of blocking (cf. Kamin, 1969), which has been frequently studied in classical conditioning paradigms in which the measure of the effect is a reduction in excitatory stimulus control. In a recent experiment performed in our laboratory (Robles, Newlin, \& Thomas, 1980) utilizing the measure of dimensional stimulus control, a manipulation that might be expected to produce blocking, that is, the superimposition on the training stimulus of an $\mathbf{S}+$ from a previous discrimination, actually resulted in an increase in dimensional stimulus control, that is, a sharper generalization gradient. In another experiment, Thomas, Burr, and Eck (1970) gave rats either discrimination training or nondifferential training and tested for control by an incidental (irrelevant) stimulus. They found that discrimination training yielded less responding to the incidental stimulus, which is consistent with the Rescorla and Wagner (1972) formulation, which is concerned only with the measure of excitatory stimulus control. Thomas et al. also measured dimensional stimulus control in this same experiment by comparing responding to the incidental stimulus with that emitted to a variation of that stimulus (a dimmer light), and they found that discrimination training had led to a sharper gradient, that is, greater dimensional control, a result inconsistent with the Blough and Mackintosh models. This result is, however, consistent with the theory of general attention (Thomas, 1970), which considers only the measure of dimensional stimulus control. Thus, the conclusion as to whether discrimination training enhances or reduces "control" by an incidental cue depends upon the measure (and thus the definition) of stimulus control employed, and, consequently, which of two "competing" theoretical interpreta- tions is supported by the data depends entirely upon which data are considered. It should be clear to all that the unitary conception of "stimulus control" as a reflection of "signal value" or of what has been learned about a stimulus has outlived its usefulness.

\section{REFERENCES}

Blough, D. S. Steady state data and a quantitative model of operant generalization and discrimination. Journal of Experimental Psychology: Animal Behavior Processes, 1975, 104, 3-21.

Hearst, E., Besley, S., \& Farthing, G. W. Inhibition and the stimulus control of operant behavior. Journal of the Experimental Analysis of Behavior, 1970, 14, 373-409.

Hearst, E., \& Koresko, M. B. Stimulus generalization and amount of prior training on variable-interval reinforcement. Journal of Comparative and Physiological Psychology, 1968, 66, 133-138.

HEARst, E., \& PoPPEN, R. Steepened generalization gradients after massed extinction to the CS. Psychonomic Science, 1965, 2, 83-84.

Hickis, C. F., Robles, L., \& Thomas, D. R. Contextual stimuli and memory retrieval in pigeons. Animal Learning \& Behavior, 1977, 5, 161-168.

Kamin, L. J. Predictability, surprise, attention, and conditioning. In B. Campbell \& R. Church (Eds.), Punishment and aversive behavior. New York: Appleton-Century-Crofts, 1969.

Mackintosh, N. J. A theory of attention: Variations in the associability of stimuli with reinforcement. Psychological Review, $1975,82,276-298$.

Mackintosh, N. J. Stimulus control: Attentional factors. In W. K. Honig \& J. E. R. Staddon (Eds.), Handbook of operant behavior. Englewood Cliffs, N.J: Prentice-Hall, 1977.

Nevin, J. A. Stimulus control. In J. A. Nevin \& G. S. Reynolds (Eds.), The study of behavior. Glenview, Ill: Scott, Foresman, 1973.

Resconla, R. A., \& W Agner, A. R. A theory of Pavlovian conditioning: Variations in the effectiveness of reinforcement and nonreinforcement. In A. H. Black \& W. F. Prokasy (Eds.), Classical conditioning II: Current research and theory. New York: Appleton-Century-Crofts, 1972.

Robles, L., Newlin, R. J., \& Thomas, D. R. Potentiation of attentional enhancement in the pigeon produced by a "blocking" stimulus. Animal Learning \& Behavior, 1980, 8, 31-36.

Schadler, M., \& Thomas, D. R. On the acquisition of dimensional stimulus control in pigeons. Journal of Comparative and Physiological Psychology, 1972, 79, 82-89.

Schw A RTz, B. Psychology of learning and behavior. New York: Norton, 1978.

Schwarz, B., \& Gamzu, E. Pavlovian control of operant behavior: An analysis of autoshaping and its implications for operant conditioning. In W. K. Honig \& J. E. R. Staddon (Eds.), Handbook of operant behavior. Englewood Cliffs, N.J: PrenticeHall, 1977.

Thомаs, D. R. Stimulus selection, attention, and related matters. In J. H. Reynierse (Ed.), Current issues in animal learning. Lincoln: University of Nebraska Press, 1970.

Thomas, D. R., Burr, D. E. S., \& Eck, K. O. Stimulus selection in animal discrimination learning: An alternative interpretation. Journal of Experimental Psychology, 1970, 86, 53-61.

Thomas, D. R., \& Kina, R. A. Stimulus generalization as a function of level of motivation. Journal of Experimental Psychology, 1959, 57, 323-328.

Weisman, R. G., \& Thio, H. Dimensional stimulus control following brief wave-length training. Journal of the Experimental Analysis of Behavior, 1976, 26, 191-197.

(Manuscript received June 7, 1981; revision accepted for publication November 16,1981 .) 